\title{
A lattice Boltzmann study of reactive microflows
}

\section{Citation}

Gabrielli, A., S. Succi, and E. Kaxiras. 2002. "A Lattice Boltzmann Study of Reactive

Microflows." Computer Physics Communications 147 (1-2): 516-21. https://doi.org/10.1016/ s0010-4655(02)00361-2.

\section{Permanent link}

http://nrs.harvard.edu/urn-3:HUL.InstRepos:41384106

\section{Terms of Use}

This article was downloaded from Harvard University's DASH repository, and is made available under the terms and conditions applicable to Other Posted Material, as set forth at http:// nrs.harvard.edu/urn-3:HUL.InstRepos:dash.current.terms-of-use\#LAA

\section{Share Your Story}

The Harvard community has made this article openly available.

Please share how this access benefits you. Submit a story.

\section{Accessibility}




\title{
A lattice Boltzmann study of reactive microflows
}

\author{
A. Gabriellii ${ }^{1}$, S. Succi ${ }^{2,3 *}$, E. Kaxiras ${ }^{3}$ \\ ${ }^{1}$ INFM, Dipartimento di Fisica, Università di Roma "La Sapienza", P.le A. Moro 2, 00185 - Roma, Italy, \\ e-mail: andrea@pil.phys.uniroma1.it \\ ${ }^{2}$ CNR, Istituto di Applicazioni Calcolo, viale Policlinico 137, 00161 - Roma, Italy \\ e-mail: succi@iac.rm.cnr.it \\ ${ }^{3 *}$ Visiting Scholar, Lyman Lab. of Physics, Harvard University, Cambridge, USA \\ ${ }^{3}$ Lyman Laboratory of Physics, Harvard University, Cambridge, USA
}

(October 30, 2018)

\begin{abstract}
The role of geometrical micro-barriers on the conversion efficiency of reactive flows in narrow threedimensional channels of millimetric size is investigated. Using a Lattice-Boltzmann-Lax-Wendroff code, we show that micro-barriers have an appreciable effect on the effective reaction efficiency of the device. If extrapolated to macroscopic scales, these effects can result in a sizeable increase of the overall reaction efficiency.
\end{abstract}

47.70.Fw, 47.11.+j

\section{INTRODUCTION}

One of the outstanding frontiers of modern applied physics/mathematics consists in the formulation of models and numerical tools for the description of complex phenomena involving multiple scales in space and time [1]. An important example of complex multiscale phenomena is the dynamics of reactive flows, a subject of wide interdisciplinary concern in theoretical and applied science. The complexity of reactive flow dynamics is parametrized by three dimensionless quantities: the Reynolds number $R e=U L / \nu$, the Damkohler number $D a=\tau_{h} / \tau_{c}$, and the Peclet number Pe $=U H / D$. Here $U, L$ and $H$ denote the macroscopic flow speed and longitudinal/transversal lengths of the flow respectively, $\nu$ is the fluid kinematic viscosity and $D$ the pollutant molecular diffusivity. The quantities $\tau_{c}$ and $\tau_{h}$ represent typical timescales of chemical and hydrodynamic phenomena. High Reynolds numbers are associated with turbulence. High Damkohler numbers imply that chemistry is much faster than hydrodynamics, so that reactions are always in chemical equilibrium and take place in tiny regions. In the opposite regime the chemistry is slow and always takes place at local mechanical equilibrium. Finally, high Peclet numbers imply that the transported species stick tightly to the fluid carrier. Varying $R e-D a-P e$ and considering different device morphologies meets with an enormous variety of chemico-physical behaviours [2]. In this work we deal with low-Reynolds, fast-reacting flows with heterogeneus catalysis. In particular we wish to gain insights into the role of geometric micro-irregularities on the effective absorption rate of tracer species at catalytic boundaries. For a detailed study see also [3].

\section{MATHEMATICAL MODEL OF REACTIVE MICROFLOW DYNAMICS}

We deal with a quasi-incompressible, isothermal flow with soluted species transported (advect and diffuse) by the flow and, upon reaching solid walls, they undergo catalytic chemical reactions. The basic equations of fluid motion are:

$$
\begin{aligned}
& \text { (i) } \partial_{t} \rho+\operatorname{div} \rho \mathbf{u}=0 \text { and (ii) } \partial_{t} \rho \mathbf{u}+\operatorname{div} \rho \mathbf{u u}= \\
& -\nabla P+\operatorname{div}\left[2 \mu\left(\nabla \mathbf{u}+(\nabla \mathbf{u})^{T}\right)+\lambda \operatorname{div} \mathbf{u}\right]
\end{aligned}
$$

where $\rho$ is the flow density, $\mathbf{u}$ the flow speed, $P=\rho T$ the fluid pressure, $T$ the temperature and $\mu, \lambda$ are the shear and bulk dynamic viscosities respectively (for the present case of quasi-incompressible flow with div $\mathbf{u} \simeq 0$ the latter can safely be ignored). Finally, uu denotes the dyadic tensor $u_{a} u_{b}, a, b=x, y, z$.

Multispecies transport with chemical reactions is described by a generalized continuity-diffusion equation for each of $s=1, \ldots, N_{s}$ species:

$$
\begin{aligned}
& \partial_{t} C_{s}+\operatorname{div} C_{s} \mathbf{u}= \\
& \operatorname{div}\left[D_{s} C_{T} \nabla\left(C_{s} / C_{T}\right)\right]+\dot{\Omega}_{s} \delta\left(\mathbf{x}-\mathbf{x}_{w}\right),
\end{aligned}
$$

where $C_{s}$ denotes the mass density of the generic $s$-th species, $D_{s}$ its mass diffusivity, $C_{T}=\sum_{s} C_{s}$ the total mass of transported species and $\dot{\Omega}_{s}$ is a chemical reaction term whose contribution is non-zero along the reactive surface described by the coordinate $\mathbf{x}_{w}(\delta(x)$ is the usual Dirac delta function). In the following the subscripts $w$ and $g$ mean "wall" (solid) and "gas" in a contact with the wall respectively.

According to Fick's law, the outgoing (bulk-to-wall) diffusive mass flux (molecules per unit surface and time) is given by (hereafter species index $s$ is omitted for simplicity):

$$
J_{g \rightarrow w}=-\left.D \partial_{\perp} C_{g}\right|_{w a l l}
$$

where $\partial_{\perp}$ denotes the normal-to-wall component of the gradient. Upon contact with solid walls, the transported species react according to the following empirical rate equation:

$$
\dot{\Omega} \equiv \frac{d C_{w}}{d t}=J_{g \rightarrow w} \frac{\Delta S}{\Delta V}-K_{c} C_{w},
$$


where $\Delta V$ is the volume element of the reactive wall and $\Delta S$ is the surface element across which fluid-wall mass transfer takes place. In our case the ratio $\Delta V / \Delta S$ is simply the thickness of the reactive wall. $K_{c}$ is the chemical reaction rate dictating species consumption once a molecule is absorbed by the wall. In the following we will use the common linear assumption

$$
J_{g \rightarrow w} \frac{\Delta S}{\Delta V}=K_{w}\left(C_{g}-C_{w}\right)
$$

where $K_{w}$ is the wall-fluid mass transfer rate. In practice each boundary cell can be regarded as a microscopic chemical reactor sustained by the mass inflow from the fluid. Chemistry (Eqs. 2, 3) sets a time-scale for the steady-state mass exchange rate. At steady state we obtain:

$$
C_{w}=\frac{K_{w}}{K_{w}+K_{c}} C_{g}
$$

Hence

$$
J_{g \rightarrow w} \frac{\Delta S}{\Delta V}=\frac{C_{g}}{\tau_{w}+\tau_{c}},
$$

where $\tau_{w}=1 / K_{w}$ and $\tau_{c}=1 / K_{c}$. These expressions show that finite-rate chemistry $\left(K_{c}>0\right)$ ensures a nonzero steady wall outflux of pollutant.

\section{THE COMPUTATIONAL METHOD}

The flow field is solved by a Lattice Boltzmann Equation (LBE) method [4] while the multispecies transport and chemical reactions are handled with a variant of the Lax-Wendroff method (LW) [8]. The LW scheme represents a numerically convenient choice recently developed to address multicomponent fluid transport (and reaction) within a LBE-like language.

\section{A. Multiscale considerations}

In this study, the unperturbed geometry of the catalytic device is a straight channel of size $L$ lattice units along the flow direction (positive $x$ direction) and $H \times H$ across it ( $y$ and $z$ axes). We add to this unperturbed geometry a single protrusion (barrier) of unitary thickness at a fixed $x=L / 2$ with height $h$ in the $z$ direction, and spanning the channel in the $y$ direction (see Fig.1).

This problem involves at least four relevant timescales. The relevant fluid scales are the advective and momentum-diffusive time:

$$
\tau_{A}=L / U \text { and } \tau_{\nu}=H^{2} / \nu,
$$

The relevant time-scales for species dynamics are:

$$
\tau_{D}=H^{2} / D, \tau_{w}=K_{w}^{-1}, \text { and } \tau_{c}=K_{c}^{-1} .
$$

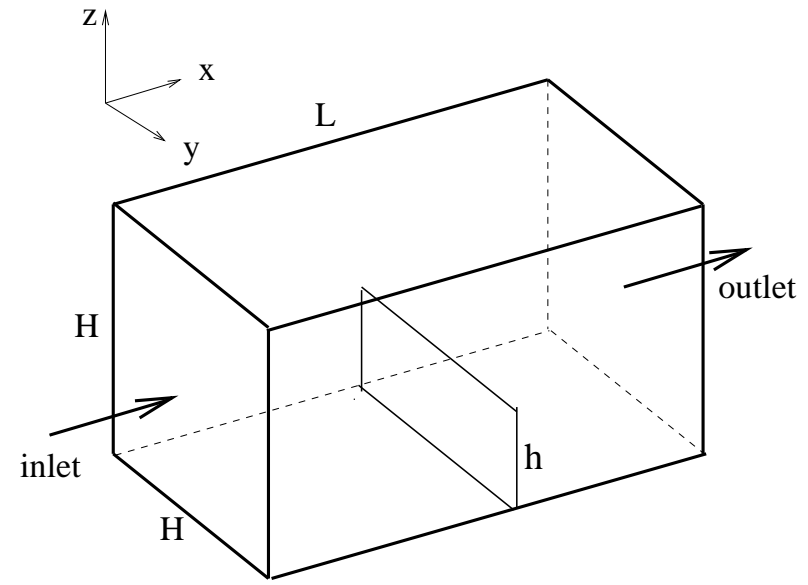

FIG. 1. Typical geometrical set up of the channel flow with a barrier on the bottom wall perpendicular to the flow of height $h$.

As discussed in the introduction, they define the major dimensionless parameters

$$
\begin{aligned}
& R e=U H / \nu \equiv \tau_{A} / \tau_{\nu}, P e=U H / D \equiv \tau_{A} / \tau_{D} \\
& D a_{c}=\tau_{c} / \tau_{A}, \quad D a_{w}=\tau_{w} / \tau_{A} .
\end{aligned}
$$

\section{CATALYTIC EFFICIENCY}

The device efficiency is defined as amount of pollutant burned per unit mass injected:

$$
\eta=\frac{\Phi_{\text {in }}-\Phi_{\text {out }}}{\Phi_{\text {in }}}
$$

where $\Phi(x)=\int[u C](x, y, z) d y d z$ is the longitudinal mass flow of the pollutant at section $x$ and $u$ is the $x$ component of $\mathbf{u}$ ( $v$ and $w$ will be the $y$ and $z$ components respectively). The in-out longitudinal flow deficit is equal to the amount $\Gamma$ of pollutant absorbed at the catalytic wall per unit time.

The goal of the optimization problem is to maximize $\Gamma$ at a given $\Phi_{i n}$. This means maximizing complex configuration-dependent quantities, such as the wall distribution of the pollutant and its normal-to-wall gradient. For future purposes, we find it convenient to recast the catalytic efficiency as $\eta=1-T$, where $T$ is the channell transmittance $T \equiv \Phi_{\text {out }} / \Phi_{\text {in }}$. Roughly speaking, in the limit of fast-chemistry, this is controlled by the ratio of advection to diffusion timescales. It is intuitive that high efficiencies are associated with large values of the ratio $\tau_{A} / \tau_{D}$, namely low-Peclet numbers.

\section{THE ROLE OF MICRO-IRREGULARITIES}

We now discuss the main qualitative effect of the micro-barrier from a microscopic point of view. 
Firstly, it provides a potential enhancement of reactivity via the increase of the surface/volume ratio. How much of this potential is actually realized depends on the resulting flow configuration.

Here, the fluid plays a two-faced role. First, geometrical restrictions lead to local fluid acceleration, hence less time for the pollutant to migrate from the bulk to the wall before being convected away by the mainstream flow. This effect may become appreciable on micro-scales for micro-flows with $h / H \simeq 0.1$ (like in actual catalytic converters). Moreover, obstacles shield away part of the active surface (wake of the obstacle) where the fluid circulates at much reduced rates (stagnation) so that less pollutant is fed into the active surface. The size of the shielded region is proportional to the Reynolds number of the flow. On the other hand, if by some mechanism the flow proves capable of feeding the shielded region, then efficient absorption is restored simply because the pollutant is confined by recirculating patterns and has almost infinite time to react without being convected away. This case is met mainly in presence of sufficiently energetic turbulent fluctuations at high values of the micro barrier-Peclet number $P e_{h}=\frac{w^{\prime} h}{D} \gg 1$ where $w^{\prime}$ is the $z$-component of the velocity field at the barrier tip.

With some appropriate approximations [3], one can show that the efficency is:

$$
\eta_{0} \simeq 1-e^{-L / l}
$$

where $l=l_{\perp}^{2} \bar{U} / D, \bar{U}=\sum_{y, z} u C / \sum_{y, z} C, l_{\perp}^{2}=$ $C \tau H^{2} /\left(2 C_{g}\right)$ and $\tau \simeq\left(1 / \tau_{D}+1 /\left(\tau_{c}+\tau_{w}\right)\right)^{-1}$.

Note that in the low absorbtion limit $L \ll l$, the above relation reduces to $\eta_{0} \simeq L / l$, meaning that halving, say, the absorption length implies same efficiency with a twice shorter catalyzer. In the opposite high-absorption limit, $L \gg l$, the relative pay-off becomes increasingly less significant.

We now turn to the case of a "perturbed" geometry. Let us begin by considering a single barrier of height $h$ (Fig. 1). The reference situation is a smooth channel at high Damkohler (Eq. 6). From [3] we find an estimate of perturbative corrections in the smallness parameter $g \equiv h / H$ :

$$
\frac{\delta \eta}{\eta_{0}}=\simeq \frac{A}{2} \frac{h}{H} R e_{h}[S c+K(a-1)]
$$

where $A=H / L$ is the aspect ratio of the channel, $S c=$ $\nu / D$ is the Schmidt number, and $a$ is a regime dependent parameter. The wake length $W$ can be estimated by $W / h=K R e_{h}$ with $K \simeq 0.1$. Three distinctive cases can be identified: (i) $a=0$ : the wake region is totally deactivated, absorption zero; (ii) $a=1$ : absorption in the wake region is exactly the same as for unperturbed flow; (iii) $a>1$ : the wake absorption is higher than with unperturbed flow (back-flowing micro-vortices can hit the rear side of the barrier).

\section{APPLICATION: REACTIVE FLOW OVER A MICROBARRIER}

The computational scheme has been applied to a fluid flowing in a millimeter-sized box of size $2 \times 1 \times 1$ millimeters along the $x, y, z$ directions with a perpendicular barrier of height $h$ (see Fig. 1). Upon using a $80 \times 40 \times 40$ grid, we obtain a lattice with $d x=d y=d z=0.0025$ $(25 \mu \mathrm{m})$. We assume a real sound speed $V_{s}=300 \mathrm{~m} / \mathrm{s}$ which becomes $c_{s}=1 / \sqrt{3}$ in lattice units. Therefore a time-step is equivalent to $d t=c_{s} d x / V_{s} \simeq 50 \mathrm{~ns}$.

The flow is forced with a constant volumetric force which mimics the effects of a pressure gradient. The fluid flow carries a passive pollutant which is continuously injected at the inlet with a flat profile across the channel. Diffusing across the flow, it reaches solid walls where it reacts according to a first order catalytic reaction: $C+A \rightarrow P$, where $A$ denote an active catalyzer and $P$ the reaction products. The initial conditions are:

$$
\begin{aligned}
& C(x, y, z)=1 \text { at the inlet, and }=0 \text { elsewhere } \\
& \rho(x, y, z)=1 \\
& u(x, y, z)=U_{0}, \quad v(x, y, z)=w(x, y, z)=0 .
\end{aligned}
$$

The pollutant is then released at the open outlet, while flow periodicity is imposed at the inlet/outlet boundaries. On the upper and lower walls, the flow speed is forced to vanish, whereas the fluid-wall mass exchange is modelled via a mass transfer rate equation of the form previously discussed. Our simulations refer to the following values (in lattice units): $U_{0} \simeq 0.1-0.2, D=0.1$, $\nu=0.01, K_{c}=K_{w}=0.1$. This implies $P e \simeq 40, \quad R e \simeq$ 400, $D a>80$ (see also Eq. (4). In order to study the effects of the barrier height $h$, we consider the following values: $h=0,2,4,8$. The typical simulation time is $t=32000$ time-steps (about 1.6 milliseconds in physical time) corresponding to two mass diffusion times across the channel. We may estimate the reference efficiency for the case of smooth channel: with $\bar{U} \simeq 0.1$, and $\tau=20$, we obtain $l \simeq 200$, hence $\eta_{0} \simeq 0.33$.

A typical two-dimensional cut of the flow pattern and pollutant spatial distribution in the section $y=H / 2$ is shown in Figs. 2 and 3, which refer to the case $h=8$.

An extended (if feeble) recirculation pattern is well visible past the barrier. Also, enhanced concentration gradients on the tip of the barrier is easily recognized from Fig. 3. The integrated concentration of the pollutant $C(x)=\sum_{y, z} C(x, y, z)$ is presented in Fig. 4 for the cases $h=0,2,4,8$. The main highlight is a substantial reduction of the pollutant concentration with increasing barrier height. We measure also the the pollutant longitudinal mass flow $\Phi(x)$. The efficiency $\eta$ is defined by Eq. 5. The results are shown in Table I1, where subscript $A$ refers to Eq. 7 with $a=1$. These results are in a reasonable agreement with the analytical estimate apart deviations $h=8$ for which the overall efficency is overestimated. Leaving aside the initial portion of the channel, 
$u(x, z)$ at $y=H / 2: t=32000$

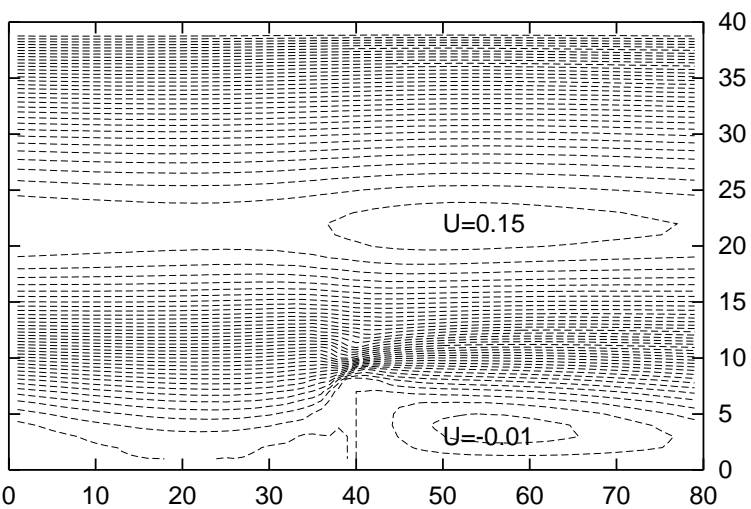

FIG. 2. Typical two-dimensional cut of the flow pattern with a single barrier of heigth $h=8$. Streamwise flow speed in the plane $y=H / 2$.

$\mathrm{C}(\mathrm{x}, \mathrm{z})$ at $\mathrm{y}=\mathrm{H} / 2: \mathrm{t}=32000$

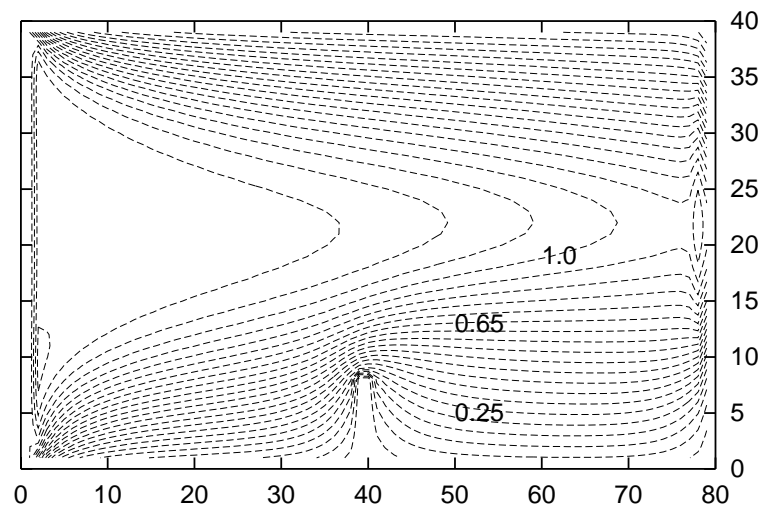

FIG. 3. Concentration isocontyurs with a single barrier of heigth $h=8$ on the plane $y=H / 2$.

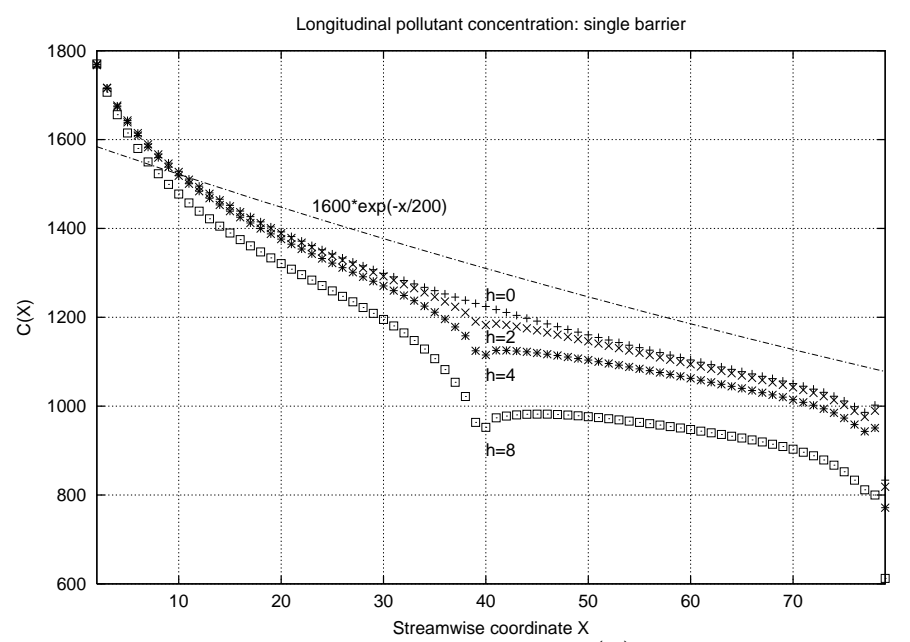

FIG. 4. Integrated concentration $C(x)$ of the pollutant with a single barrier of height $h=0,2,4,8$ after 32000 steps. The dashed line represent a theoretical evaluation with no barrier $(h=0)$ and $l \simeq 200$.

\begin{tabular}{|l|c|c|c|l}
\hline \hline Run & $h / H$ & $\eta$ & $\frac{\delta \eta}{\eta}, \frac{\delta \eta_{A}}{\eta_{A}}$ & \\
\hline R00 & 0 & 0.295 & 0.00 & \\
\hline R02 & $1 / 20$ & 0.301 & $0.02,0.025$ & \\
\hline R04 & $1 / 10$ & 0.312 & $0.06,0.10$ & \\
\hline R08 & $2 / 10$ & 0.360 & $0.22,0.40$ & \\
\hline \hline
\end{tabular}

TABLE I. Single barrier at $x=40$ : the effect of barrier Z height.

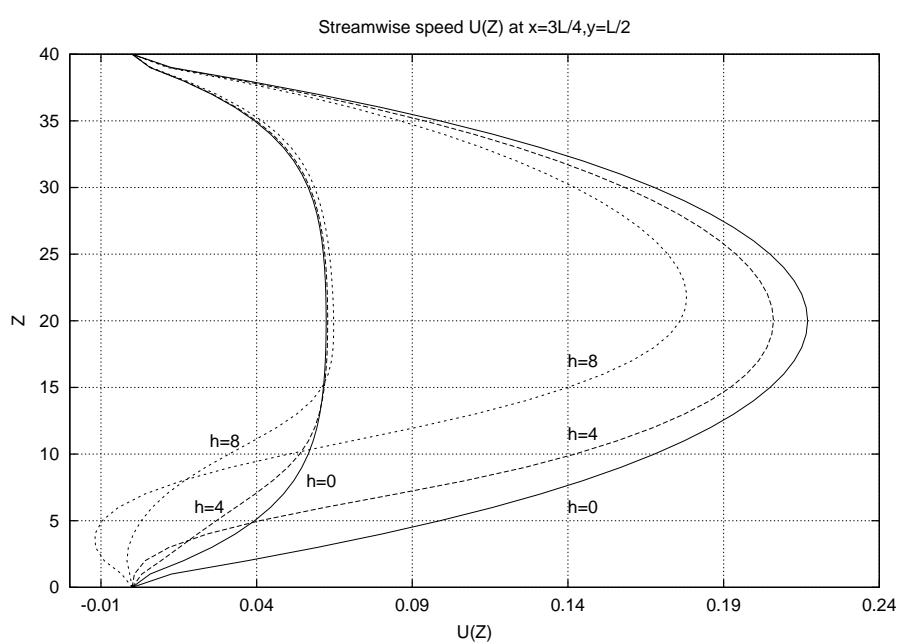

FIG. 5. Time evolution of the transversal streamwise speed $u(z)$ at $x=3 L / 4$ and $y=L / 2$. Single barrier of varying zheight $h=0,4,8$ at $t=3200$ and $t=32000$. Note the backflow for $h=8$ at small $z$.

our numerical data are pretty well fitted by an exponential with absorption length $l=200$, in a good agreement with the theoretical estimate $l \simeq 200$. The barrier also promotes a potentially beneficial flow recirculation, which is well visible in Figs. 5 and 6 . They clearly reveal a recirculating backflow for $h=8$. For applications to many barriers see [3].

\section{UPSCALING TO MACROSCOPIC DEVICES}

It is important to realize that even tiny improvements on the microscopic scale can result in pretty sizeable cumulative effects on the macroscopic scale of the real devices, say 10 centimeters. The efficiency of an array of $N$ serial micro-channels can be estimated simply as

$$
\eta_{N}=1-T^{N} .
$$

It is readily recognized that even low single-channel efficiencies can result in significant efficiencies of macroscopic devices with $N=10-100$ (see Fig. 7). Equation 8 with numerical data from present simulations provide satisfactory agreement with experimental data [9, 10 .

Nonetheless, extrapolations based on Eq. 8 must be taken very cautiously in the case of rugh or fractal walls [11] or of fully developed turbulence. 


\section{CONCLUSIONS}

Stream function at $y=L / 2: h=8,160000$ timesteps

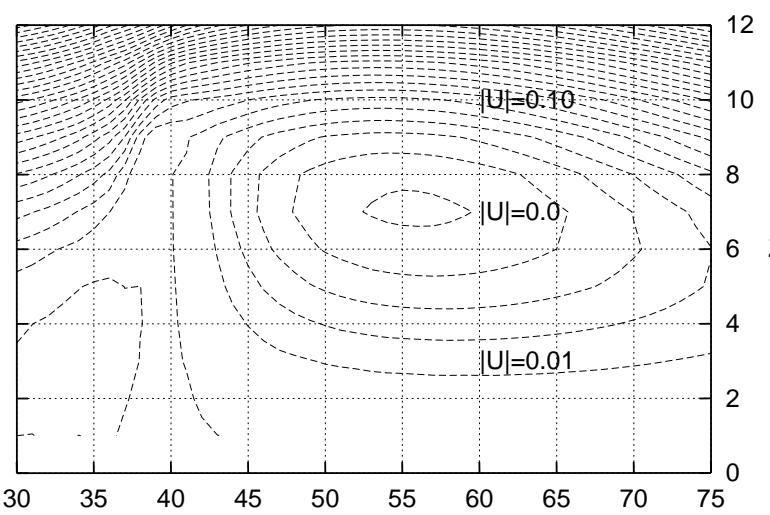

FIG. 6. Blow-up of the streaxnlines of the flow field past a barrier of height $h=8$ located at $x=40$. The velocity direction in the closed streamlines of the vortex is clockwise. The recirculation effects are feeble and depletion is dominant. In fact for $h=8$ the local Peclet number is $\sim 0.01 \cdot 8 / 0.1=0.8$, seemingly too small to provide micro-turbulent effects.

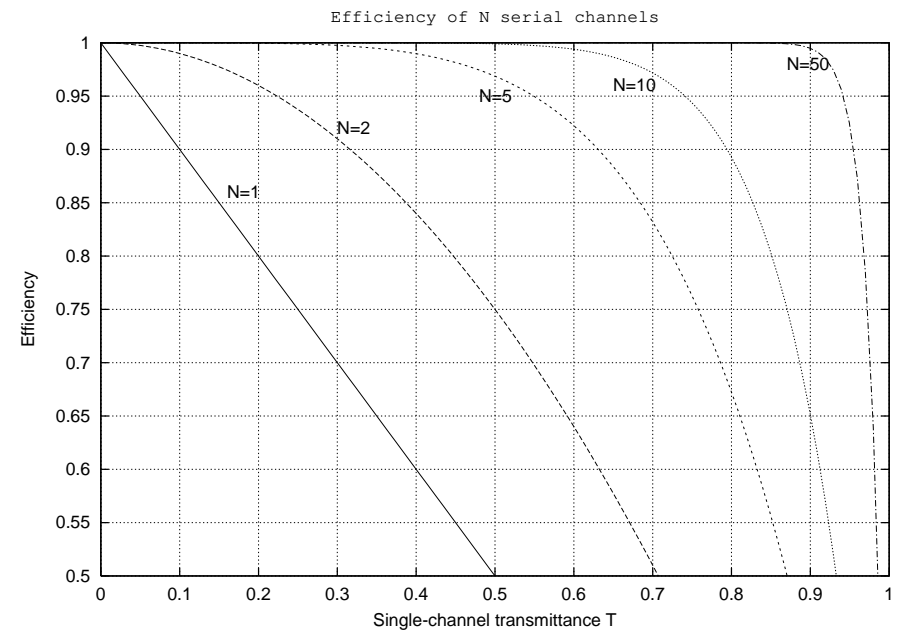

FIG. 7. Efficiency of a series of $N$ micro-channels as a function of the single-channel transmittance.
Although these simulations generally confirm qualitative expectations on the overall dependence on the major physical parameters, they also highlight the existence of non-perturbative effects, such as the onset of microvorticity in the wake of geometrical obstrusions, which are hardly amenable to analytical treatment.

Work performed under NATO Grant PST.CLG. 976357.

[1] F. Abraham, J. Broughton, N. Bernstein, E. Kaxiras, Comp. in Phys., 12, 538 (1998).

[2] E. Oran, J. Boris, Numerical simulation of reactive flows, Elsevier Science, New York, 1987.

[3] S. Succi, A. Gabrielli, G. Smith, and E. Kaxiras, Euro. Phys. Jour. Appl. Phys. to appear, physics/0103041 on http://xxx.lanl.gov.

[4] G. Mc Namara, G. Zanetti, Phys. Rev. Lett., 61, 2332 (1988).

[5] F. Higuera, S. Succi, R. Benzi, Europhys. Lett., 9, 345 (1989).

[6] R. Benzi, S. Succi and M. Vergassola, Phys. Rep., 222, 145 (1992).

[7] Y. Qian, D.d'Humieres, P. Lallemand, Europhys. Lett., 17, 149 (1989).

[8] S. Succi, G. Bella, H. Chen, K. Molvig, C. Teixeira, J. Comp. Phys., 152, 493 (1999).

[9] S. Succi, G. Smith, E. Kaxiras, J. Stat. Phys., 2001, submitted.

[10] A. Bergmann, R. Bruck, C. Kruse, Society of Automotive Engineers (SAE) technical paper SAE 971027, Proceedings of the 1997 International SAE Congress, Detroit, USA, February 1997.

[11] J. S. Andrade Jr, M. Filoche, and B. Sapoval, Europhys. Lett. to appear, cond-mat/0012258 on http://xxx.lanl.gov. 